04,05,09

\title{
Отрицательный сдвиг Гуса-Хенхена на границе многоподрешеточного антиферродиэлектрика
}

\author{
() В.Д. Пойманов ${ }^{1}$, В.Г. Шавров ${ }^{2}$ \\ ${ }^{1}$ Донецкий национальный университет, \\ Донецк, Украина \\ ${ }^{2}$ Институт радиотехники и электроники им. В.А. Котельникова РАН, \\ Москва, Россия \\ E-mail: Vladislav.Poymanow@yandex.ru
}

Поступила в Редакцию 30 декабря 2019 г.

В окончательной редакции 30 декабря 2019 г.

Принята к публикации 10 января 2020 г.

Показано, что взаимодействие электромагнитного излучения с электродипольноактивными спиновыми модами в многоподрешеточном антиферродиэлектрике может приводить к аномальному (отрицательному) пространственному сдвигу светового пучка при падении электромагнитной волны на границу раздела с изотропной диэлектрической средой.

Ключевые слова: поверхностные поляритоны, отрицательная рефракция. Сдвиг Гуса-Хэнхена, электродипольноактивный антиферродиэлектрик.

DOI: $10.21883 /$ FTT.2020.05.49228.08M

В настоящее время большое количество работ посвящено изучению взаимодействия электромагнитного излучения с собственными колебаниями спиновой подсистемы многоподрешеточного магнетика [1-10]. В этом случае многообразие магнитных и оптических свойств обусловлено, в частности, отсутствием центральносимметричности в такой системе [3]. Например, гематит $\alpha-\mathrm{Fe}_{2} \mathrm{O}_{3}$ содержит четыре подрешетки, соответственно чему колебания спиновой подсистемы описываются четырьмя независимыми векторами, представляющими собой линейные комбинации векторов намагниченности отдельных подрешеток [11]. Таким образом, в спектре собственных колебаний гематита имеется четыре ветви, две из которых являются акустическими магнитоактивными модами с характерными частотами в инфракрасной области спектра. Две других являются обменными электродипольноактивными возбуждениями и могут наблюдаться в экспериментах по неупругому рассеянию нейтронов [9].

Настоящая работа посвящена изучению взаимодействия электромагнитной волны (ЭМВ) с обменными модами антиферродиэлектрика (АФД). Ее целью будет изучение особенностей отражения ТМ-поляризованной волны от структуры, содержащей АФД (среда 1). В частности, рассмотрим эффект, пространственного сдвига пучка ЭМВ относительно точки падения вдоль границы раздела (сдвиг Гуса-Хэншена, $($ СГХ)), возникающий при полном внутреннем отражении при падении ЭМВ из диэлектрика (среда 2). Ввиду отсутствия плазмонных ветвей в спектре собственных колебаний среды 2, ее диэлектрическую проницаемость будем считать скалярной величиной, равной $\varepsilon_{0}$.
В [11] было получено, что гамильтониан вышеуказанного взаимодействия имеет вид

$$
w=w_{0}+w_{E}
$$

и состоит из чисто магнитной части

$$
\begin{aligned}
w_{0}= & 4 M_{s}\left(\frac { 1 } { 2 } \left(-H_{e 1} l_{1}^{2}+H_{e 2} l_{2}^{2}+H_{e 3} l_{3}^{2}+H_{e 0} m^{2}\right.\right. \\
& \left.+H_{a 1} l_{1 z}^{2}+H_{a 2} l_{2 z}^{2}+H_{a 3} l_{3 z}^{2}+H_{a 0} m_{z}^{2}\right) \\
& \left.+H_{D}\left(m_{y} l_{1 x}-m_{x} l_{1 y}\right)+H_{D}^{*}\left(l_{2 x} l_{3 y}-l_{2 y} l_{3 x}\right)\right)
\end{aligned}
$$

и описывающей взаимодействие электромагнитной волны со спиновой подсистемой:

$$
\begin{aligned}
w_{E}= & \mathbf{E P}=4 M_{s}\left(E _ { x } \left[R_{3}\left(l_{1 z} l_{3 y}-l_{1 y} l_{3 z}\right)\right.\right. \\
& \left.+R_{2}\left(l_{1 z} l_{2 x}-l_{1 x} l_{2 z}\right)+r_{3}\left(m_{x} l_{3 z}-m_{z} l_{3 x}\right)\right] \\
& +E_{y}\left[R_{3}\left(l_{1 x} l_{3 z}-l_{1 z} l_{3 x}\right)+R_{2}\left(l_{1 z} l_{2 y}-l_{1 y} l_{2 z}\right)\right. \\
& \left.+r_{3}\left(m_{y} l_{3 z}-m_{z} l_{3 y}\right)\right]+E_{z}\left[\pi_{1} \mathbf{m l}_{3}+\pi_{2} \mathbf{l}_{1} \mathbf{l}_{2}\right. \\
& \left.\left.+R_{3 z}\left(l_{1 y} l_{3 x}-l_{1 x} l_{3 y}\right)+r_{2 z}\left(m_{x} l_{2 y}-m_{y} l_{2 x}\right)\right]\right) .
\end{aligned}
$$

Здесь

$$
\mathbf{m}=\mathbf{m}_{1}+\mathbf{m}_{2}+\mathbf{m}_{3}+\mathbf{m}_{4}, \quad \mathbf{l}_{1}=\mathbf{m}_{1}-\mathbf{m}_{2}+\mathbf{m}_{3}-\mathbf{m}_{4},
$$

$\mathbf{l}_{2}=\mathbf{m}_{1}+\mathbf{m}_{2}-\mathbf{m}_{3}-\mathbf{m}_{4}, \quad \mathbf{l}_{3}=\mathbf{m}_{1}-\mathbf{m}_{2}-\mathbf{m}_{3}+\mathbf{m}_{4}$

- линейные комбинации намагниченностей подрешеток, $H_{e i}$ - обменные поля, $H_{a i}$ - поля анизотропии, 
$H_{D}$ - поле Дзялошинского. Гамильтониан (1) приводит к резонансной частотной зависимости компонент тензора диэлектрической проницаемости.

В работе рассмотрим две модели:

1. Сверхрешетка, компонентами которой являются АФД и диэлектрик в отсутствие внешнего поля;

2. Однородный АФД во внешнем магнитном поле $H_{0}$ приложенном вдоль вектора антиферромагнетизма $\mathbf{l}_{1}$.

В обоих случаях полагаем, что АФД в основном состоянии находится в легкоосной фазе $\left(l_{1 z}=1, l_{1 x}=l_{1 y}=0, \mathbf{m}=\mathbf{l}_{2}=\mathbf{l}_{3}=0\right)$ и является немагнитным $(\mu=1)$. Таким образом - АФД можно считать правосторонней средой.

Рассмотрим геометрию, в которой плоскостью падения является $x y$, падающая ТМ-волна поляризована вдоль $o z$, совпадающем с направлением приложения внешнего поля в случае 2. Нормаль к границе раздела направлена вдоль оси $x$. Тогда тензор диэлектрической проницаемости в общем случае можно записать как:

$$
\hat{\varepsilon}_{a}=\left(\begin{array}{ccc}
\varepsilon_{1} & i \varepsilon^{\prime} & 0 \\
-i \varepsilon^{\prime} & \varepsilon_{2} & 0 \\
0 & 0 & \varepsilon_{3}
\end{array}\right)
$$

Соответственно этому поле во всем пространстве ищем в виде

$$
H_{z}(x, y)=e^{i k y}\left(\begin{array}{c}
e^{i k_{0} x}+R e^{-i k_{0} x} \\
T e^{-q x}
\end{array}\right), \quad \begin{aligned}
& x>0 \\
& x<0
\end{aligned},
$$

где буквами $R$ и $T$ обозначены френелевские коэффициенты отражения и прохождения ЭМВ соответственно. Спектр нормальных поляритонов (НП) в каждой среде, следующий из уравнений Максвелла

$$
k_{0}=\sqrt{\varepsilon_{0} \frac{\omega^{2}}{c^{2}}-k^{2}}, \quad q=\sqrt{\frac{\varepsilon_{2}}{\varepsilon_{1}} k^{2}-\left(\varepsilon_{2}-\frac{\varepsilon^{\prime 2}}{\varepsilon_{1}}\right) \frac{\omega^{2}}{c^{2}}} .
$$

Полагаем, что $k_{0}^{2}>0, q^{2}>0$, так что имеет место полное внутреннее отражение. При $x=0$ непрерывными на границе раздела являются $H_{z}$ и

$$
E_{y}=-\frac{c}{\omega}\left(\frac{i \varepsilon_{1} \frac{\partial}{\partial x}+\varepsilon^{\prime} \frac{\partial}{\partial y}}{\varepsilon_{1} \varepsilon_{2}-\varepsilon^{\prime 2}}\right) H_{z} .
$$

Записывая систему граничных условий для (6), получим коэффициент отражения в виде

$$
R=e^{-i \varphi}, \quad \varphi=2 \operatorname{arctg}\left(\frac{\varepsilon_{0}\left(\varepsilon_{1} q-\varepsilon^{\prime} k\right)}{k_{0}\left(\varepsilon_{1} \varepsilon_{2}-\varepsilon^{\prime 2}\right)}\right) .
$$

Используя (9), можно найти величину СГХ [10]

$$
\begin{aligned}
\Delta= & \frac{d \varphi}{d k}=\frac{2 \varepsilon_{0}^{2} \omega^{2}}{q k_{0}\left(k_{0}^{2}+\left(\frac{\varepsilon_{0}\left(\varepsilon_{1} q-\varepsilon^{\prime} k\right)}{\varepsilon_{1} \varepsilon_{2}-\varepsilon^{\prime 2}}\right)^{2}\right)} \\
& \times\left(k\left(\frac{\varepsilon_{2}}{\varepsilon_{1} \varepsilon_{2}-\varepsilon^{\prime 2}}-\frac{1}{\varepsilon_{0}}\right)-q \frac{\varepsilon^{\prime}}{\varepsilon_{1} \varepsilon_{2}-\varepsilon^{\prime 2}}\right) .
\end{aligned}
$$

Случай 1. В отсутствие внешнего поля ТДП этих слоев имеют вид:

$$
\hat{\varepsilon}_{k}=\left(\begin{array}{ccc}
\varepsilon_{x k} & 0 & 0 \\
0 & \varepsilon_{y k} & 0 \\
0 & 0 & \varepsilon_{z k}
\end{array}\right), \quad k=1,2
$$

причем для АФД [11]

$$
\varepsilon_{x a}(\omega)=\varepsilon_{y a}(\omega)=\varepsilon_{\perp a}(\omega)=\varepsilon_{\perp} \frac{\omega^{2}-\omega_{\varepsilon}^{2}}{\omega^{2}-\omega_{0}^{2}},
$$

где $\omega_{0}$ - частота антиферроэлектрического резонанса. Для диэлектрического слоя

$$
\left(\varepsilon_{d}\right)_{i k}=\varepsilon_{0} \delta_{i k}
$$

Ненулевые компоненты усредненного ТДП (5), полученные методом эффективной среды $[6,7]$ :

$$
\varepsilon_{1}=\frac{1}{\frac{\nu_{a}}{\varepsilon_{x a}}+\frac{\nu_{d}}{\varepsilon_{x d}}}, \quad \varepsilon_{2}=v_{a} \varepsilon_{y a}+v_{d} \varepsilon_{y d},
$$

где $v_{a}$ и $v_{d}$ - относительные толщины антиферроэлектрического и диэлектрического слоев соответственно. Подставляя сюда (12) и (13), находим

$$
\varepsilon_{1}=\varepsilon_{1}^{(0)} \frac{\omega^{2}-\omega_{\varepsilon}^{2}}{\omega^{2}-\omega_{1}^{2}}, \quad \varepsilon_{2}=\varepsilon_{2}^{(0)} \frac{\omega^{2}-\omega_{2}^{2}}{\omega^{2}-\omega_{0}^{2}} .
$$

где

$$
\varepsilon_{1}^{(0)}=\frac{\varepsilon_{0} \varepsilon_{\perp}}{v_{d} \varepsilon_{\perp}+v_{a} \varepsilon_{0}}, \quad \varepsilon_{2}^{(0)}=v_{a} \varepsilon_{\perp}+v_{d} \varepsilon_{0}
$$

$\omega_{1}^{2}=\omega_{0}^{2}+\frac{v_{d} \varepsilon_{\perp}}{v_{d} \varepsilon_{\perp}+v_{a} \varepsilon_{0}} \omega_{a}^{2}, \quad \omega_{2}^{2}=\omega_{0}^{2}+\frac{v_{a} \varepsilon_{\perp}}{v_{a} \varepsilon_{\perp}+v_{d} \varepsilon_{0}} \omega_{a}^{2}$.

Обратим внимание на то, что при $v_{a}>v_{d}$ выполняется соотношение $\omega_{2}>\omega_{1}$ и наоборот. Выражения (7) и (10) для спектра нормальных поляритонов и СГХ в сверхрешетке дают соответственно

$$
\begin{gathered}
q=\sqrt{\varepsilon_{2}\left(\frac{k^{2}}{\varepsilon_{1}}-\frac{\omega^{2}}{c^{2}}\right)}, \\
\Delta=\frac{2 \varepsilon_{0}^{2} \varepsilon_{2}^{2} \omega^{2} k}{q k_{0}\left(\left(\varepsilon_{2} k_{0}\right)^{2}+\left(\varepsilon_{0} q\right)^{2}\right)}\left(\frac{1}{\varepsilon_{1}}-\frac{1}{\varepsilon_{0}}\right) .
\end{gathered}
$$

Зависимость СГХ в этом случае на $k \omega$ - диаграмме изображена на рис. 1.

Случай 2. Однородный АФД является изотропным в плоскости падения, поэтому ТДП при наличии внешнего поля запишется в виде [9]:

$$
\hat{\varepsilon}_{a}=\left(\begin{array}{ccc}
\varepsilon_{\perp a} & i \varepsilon_{a}^{\prime} & 0 \\
-i \varepsilon_{a}^{\prime} & \varepsilon_{\perp a} & 0 \\
0 & 0 & \varepsilon_{\| a}
\end{array}\right)
$$



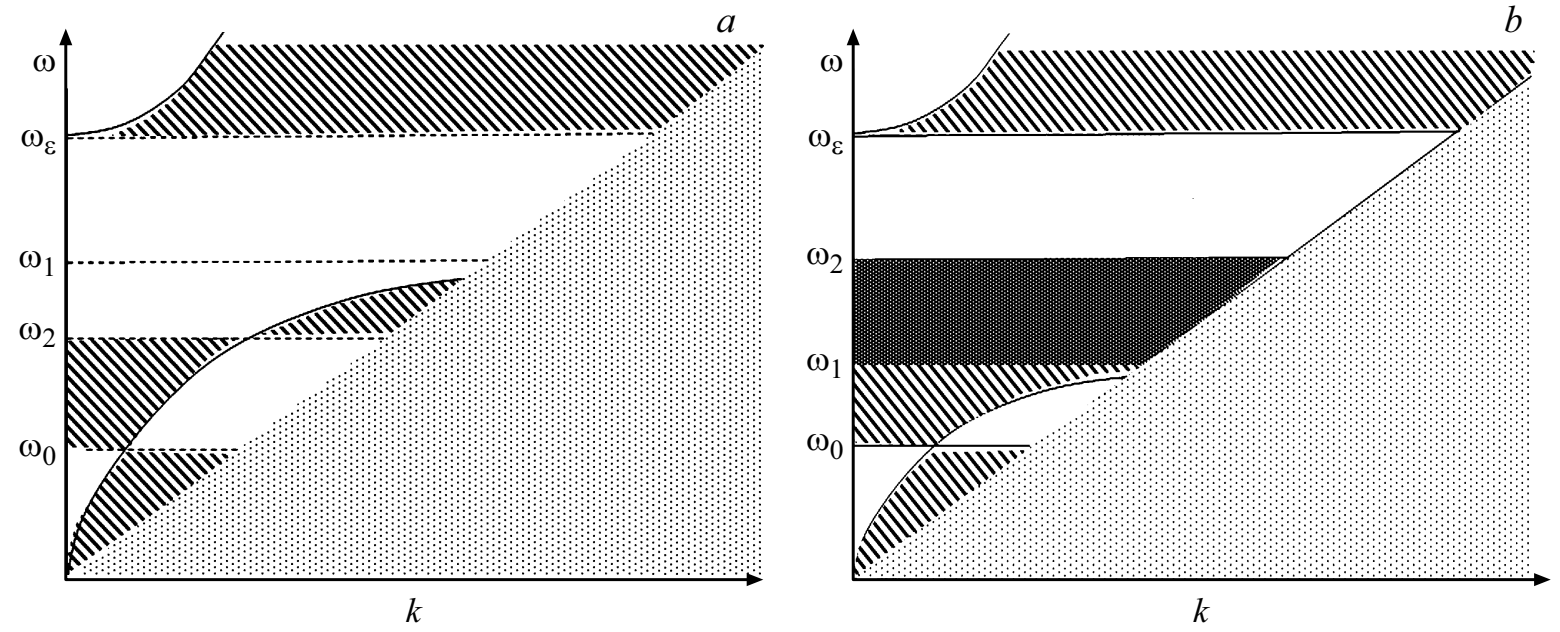

Рис. 1. Сдвиг Гуса-Хеншена для случая сверхрешетки при $v_{a}<v_{d}$ (рис. $\left.1, a\right)$ и при $v_{a}>v_{d}$ (рис. $\left.1, b\right)$. Область ниже наклонной прямой $\omega=\frac{k}{\sqrt{\varepsilon_{0}}}$ соответствует поверхностной волне в диэлектрике, косой штриховки - положительному СГХ, темная область отрицательному. Незаштрихованная область соответствует объемным волнам в АФД. Области разделены кривой $q=0$.

где

$$
\begin{gathered}
\varepsilon_{\perp a}(\omega)=\varepsilon_{\perp} \frac{\left(\omega^{2}-\tilde{\omega}_{+}^{2}\right)\left(\omega^{2}-\tilde{\omega}_{-}^{2}\right)}{\left(\omega^{2}-\omega_{0+}^{2}\right)\left(\omega^{2}-\omega_{0-}^{2}\right)} \\
\varepsilon_{a}^{\prime}(\omega)=\varepsilon_{\perp} \frac{2 \omega \omega_{H} \omega_{a}^{2}}{\left(\omega^{2}-\omega_{0+}^{2}\right)\left(\omega^{2}-\omega_{0-}^{2}\right)} \\
\omega_{0 \pm}=\omega_{0} \pm \omega_{H} \\
\tilde{\omega}_{ \pm}^{2}=\omega_{0}^{2}+\frac{\omega_{a}^{2}}{2}+\omega_{H}^{2} \pm \sqrt{4 \omega_{H}^{2}\left(\omega_{0}^{2}+\frac{\omega_{a}^{2}}{2}\right)+\left(\frac{\omega_{a}^{2}}{2}\right)^{2}}
\end{gathered}
$$

Предполагается, что внешнее поле не превышает поля спин-флоп перехода. Представим спектр нормальных поляритонов и СГХ в виде

$$
\begin{gathered}
q=\sqrt{k^{2}-\tilde{\varepsilon} \frac{\omega^{2}}{c^{2}}}, \\
\Delta=\frac{2 \varepsilon_{0}^{2} \omega^{2} \tilde{\varepsilon}^{2}}{q k_{0}\left(\left(\tilde{\varepsilon} k_{0}\right)^{2}+\left(\varepsilon_{0} \varepsilon_{\perp a}(q-\delta k)\right)^{2}\right)}\left(k\left(\frac{1}{\tilde{\varepsilon}}-\frac{1}{\varepsilon_{0}}\right)-q \frac{\delta}{\tilde{\varepsilon}}\right),
\end{gathered}
$$

где $\tilde{\varepsilon}=\varepsilon_{\perp a}\left(1-\delta^{2}\right), \delta=\frac{\varepsilon_{a}^{\prime}}{\varepsilon_{\perp a}}$.

Используя (19) и (20), представим последние выражения в виде

$$
\begin{gathered}
\tilde{\varepsilon}=\varepsilon_{\perp a} \frac{\left(\omega^{2}-\omega_{\varepsilon+}^{2}\right)\left(\omega^{2}-\omega_{\varepsilon-}^{2}\right)}{\left(\omega^{2}-\tilde{\omega}_{+}^{2}\right)\left(\omega^{2}-\tilde{\omega}_{-}^{2}\right)} \\
\delta=\frac{2 \omega \omega_{H} \omega_{a}^{2}}{\left(\omega^{2}-\tilde{\omega}_{+}^{2}\right)\left(\omega^{2}-\tilde{\omega}_{-}^{2}\right)}
\end{gathered}
$$

где $\omega_{\varepsilon \pm}=\omega_{\varepsilon} \pm \omega_{H}$.

Таким образом, знак СГХ в (21) определяется величиной $k\left(\frac{1}{\tilde{\varepsilon}}-\frac{1}{\varepsilon_{0}}\right)-q \frac{\delta}{\tilde{\varepsilon}}$.
При $\tilde{\varepsilon}>0$ условие полного внутреннего отражения дает $\tilde{\varepsilon}<\varepsilon_{0}$. Поэтому слагаемое $1 / \varepsilon_{0}$ во всяком случае не влияет на знак выражения $k\left(1 / \tilde{\varepsilon}-1 / \varepsilon_{0}\right)$. Полагаем, что $\varepsilon_{0} \gg \tilde{\varepsilon}$, что фактически несправедливо только вблизи полюсов $\tilde{\varepsilon}$. В остальных случаях СГХ можно представить в более простом виде

$$
\Delta=\frac{2 \omega}{c \sqrt{\varepsilon_{0}}(q-\delta k)^{2}}\left\{\tilde{\varepsilon}\left(\frac{k}{q}-\delta\right)\right\} .
$$

Как следует из (23), наличие внешнего поля сдвигает величину СГХ в сторону отрицательных значений. Сле-

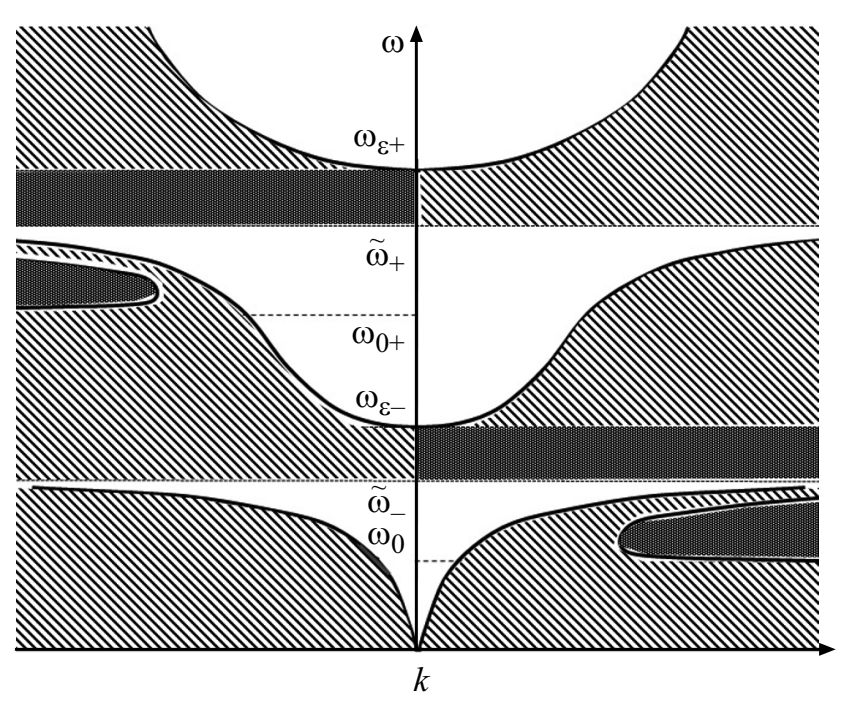

Рис. 2. Сдвиг Гуса-Хенхена для случая для однородного антиферродиэлектрика во внешнем магнитном поле. Темная область соответствует отрицательному СГХ, область косой штриховки - положительному Незаштрихованная область соответствует объемным волнам в АФД. Области разделены кривой $q=0$. 
дует обратить внимание на упорядочение характерных частот

$$
\omega_{0-}<\tilde{\omega}_{-}<\omega_{\varepsilon_{-}}<\omega_{0+}<\tilde{\omega}_{+}<\omega_{\varepsilon+}
$$

при выполнении условия $\omega_{H}<\frac{\omega_{\varepsilon}-\omega_{0}}{2}$ (в противном случае изменится соотношение между $\omega_{\varepsilon-}$ и $\left.\omega_{0+}\right)$. Характерные области для этого случая изображены на рис. 2.

Очевидно, внешнее поле также приводит к формированию областей с отрицательным СГХ. Кроме того, в этом случае направления падающей волны $k$ и $-k$ становятся неэквивалентными, т.е. в спектре наблюдается невзаимность.

Таким образом - в работе рассмотрен эффект пространственного сдвига пучка электромагнитных волн при падении на многоподрешеточный антиферродиэлектрик (Сдвиг Гуса-Хэншена). Найдены области положительного и отрицательного СГХ для случаев двухкомпонентной сверхрешетки, в состав которой входит АФД, и однородного АФД во внешнем магнитном поле, приложенном вдоль вектора антиферромагнетизма. Показано, что для формирования области отрицательного СГХ в случае сверхрешетки необходимо, чтобы толщина антиферроэлектрического слоя была больше толщины диэлектрического. В случае однородного антиферродиэлектрика указанные области формируются приложением внешнего магнитного поля, меньшего поля спинфлоп перехода. Обнаружена невзаимность спектра по отношению к направлению распространения прямой и обратной волны. Показано существование аномальных областей отрицательного СГХ.

\section{Финансирование работы}

Работа выполнена в рамках госзадания при частичном финансировании РФФИ, грант 18-07-01320.

\section{Конфликт интересов}

Авторы заявляют, что у них нет конфликта интересов.

\section{Список литературы}

[1] А.П. Пятаков, А.К. Звездин. УФН 182, 6, 593, (2012).

[2] Е.А. Туров, А.В. Колчанов, В.В. Меньшенин, И.Ф. Мирсаев, В.В. Николаев. Симметрия и физические свойства антиферромагнетиков. Физматлит, М. (2001). 560 с.

[3] Д.В. Кулагин, А.С. Савченко, С.В, Тарасенко. ЖЭТФ 133, 5,1051 (2008).

[4] А.С. Савченко, С.В. Тарасенко, Т.Н. Тарасенко. ФТТ 2, 267 (2007).

[5] А.С. Савченко, А.С. Тарасенко, С.В. Тарасенко, В.Г. Шавров. ПЖЭТФ 2, 7, 380 (2015).

[6] R.H. Tarkhanyan, D.G. Niarchos. JMMM 312, 6 (2007).

[7] R.H. Tarkhanyan, D.G. Niarchos, M. Kafesaki. JMMM 322, $603(2010)$.
[8] Е.А. Туров, В.В. Николаев. УФН 175, 5, 457 (2005).

[9] В.Н. Криворучко, Т.Е. Примак. ФТТ 33, 11, 3205 (1991).

[10] R. Macedo, R.L. Stamps, T. Dumelov. Opt. Soc. Am. 22, 23, 28467 (2014).

[11] V. N. Kruvoruchko, D.A. Yablonskii. Sov. Phys. JETP 67, 9, 1886 (1988).

Редактор Т.Н. Василевская 